\title{
Practical Methodology for the Inclusion of Nonlinear Slosh Damping in the Stability Analysis of Liquid-propelled Space Vehicles*
}

\author{
John A. Ottander, Robert A. Hall and Joseph F. Powers
}

\begin{abstract}
One of the challenges of developing flight control systems for liquid-propelled space vehicles is ensuring stability and performance in the presence of parasitic minimally damped slosh dynamics in the liquid propellants. This can be especially difficult when the fundamental frequencies of the slosh motions are in proximity to the frequency used for vehicle control. The challenge is partially alleviated since the energy dissipation and effective damping in the slosh modes increases with amplitude. However, traditional launch vehicle control design methodology is performed with linearized systems using a fixed slosh damping corresponding to a slosh motion amplitude based on heritage values. This papers presents a method for performing the control design and analysis using damping at slosh amplitudes chosen based on the resulting limit cycle amplitude of the vehicle thrust vector system due to a control-slosh interaction under degraded phase and gain margin conditions.
\end{abstract}

\section{NOMENCLATURE}

$Z \quad=$ displacement of vehicle c.g. normal to

reference trajectory $(\mathrm{m})$

$Z_{s j} \quad=$ sloshing fluid displacement in jth tank $(\mathrm{m})$

$\beta_{E} \quad=$ engine angle $(\mathrm{rad})$

$\phi \quad=$ angle of vehicle centerline $(\mathrm{rad})$

$a_{0} \quad=$ attitude control gain (-)

$a_{1} \quad=$ attitude-rate control gain (-)

$c_{2} \quad=R^{\prime} X_{\text {c.g. }} / I\left(1 / \mathrm{s}^{2}\right)$

$\mathrm{D} \quad=\operatorname{drag}$ force $(\mathrm{N})$

$F \quad=$ total engine thrust $(\mathrm{N})$

I = pitch-yaw vehicle moment of inertia including

engines and sloshing fluid $\left(\mathrm{kg}-\mathrm{m}^{2}\right)$

$k_{3} \quad=F / M\left(\mathrm{~m} / \mathrm{rad}-\mathrm{s}^{2}\right)$

$k_{4} \quad=R^{\prime} / M\left(\mathrm{~m} / \mathrm{rad}-\mathrm{s}^{2}\right)$

$l_{s j} \quad=$ c.g.-to-slosh mass distance $=X_{c . g .}-X_{s j}(\mathrm{~m})$

$l_{\text {c.p. }} \quad=$ center of percussion $=I_{x x} /\left(M * X_{\text {c.g. }}\right)(\mathrm{m})$

$M \quad$ = vehicle mass including engines and sloshing

fluid $(\mathrm{kg})$

$m_{s j} \quad=$ slosh mass, jth tank $(\mathrm{kg})$

$R^{\prime} \quad=$ vectored engine thrust $(\mathrm{N})$

$X_{\text {c.g. }} \quad=$ center of gravity measured from gimbal $(\mathrm{m})$

$X_{s j} \quad=$ slosh mass location measured from gimbal $(\mathrm{m})$

$\zeta_{s j} \quad=$ slosh damping, jth tank (-)

$\omega_{s j} \quad=$ slosh natural frequency, jth tank $(\mathrm{rad} / \mathrm{s})$

*Research supported by NASA contract number NNM12AA41C

J.A. Ottander is with Dynamics Concepts Inc., Jacobs ESSSA Group, Huntsville, AL 35806 USA (phone: 256-544-0038; email: john.a.ottander@nasa.gov)

\section{INTRODUCTION}

Liquid propelled space vehicles, especially the stages of launch vehicles, are often over $90 \%$ propellant by mass. This high propellant mass fraction is necessary in order maximize the payload that can be delivered to the desired trajectory state. The motion of the liquid propellant inside of the tanks can significantly affect the flight control stability and performance as most recently demonstrated by the failure of Flight 2 of the Space X Falcon 1 rocket in 2007 (Bjelde [7]). During Flight 2, the first stage burn was successful but approximately 90 s into the burn of the second stage, a limit cycle developed in the pitch and yaw axis due to control interaction with the liquid oxygen tank which lead to pre-mature engine shutdown. The corrective action after Flight 2, was to add ring slosh baffles to the tank to increase the damping of the slosh mode. Tank baffles are an effective way of increasing slosh damping and were used on both the Saturn V and the Space Shuttle, but the mass impact necessitates minimizing the size and number of baffles.

For flight control, the primary liquid motion of concern is the first lateral slosh mode of the liquid surface. As described in (Dodge [5]) this motion can be described with a mechanical model of either a spring-mass-damper or a pendulum with viscous friction attached at a certain location inside the tank. Just as the pendulum model natural frequency $\left(\omega_{n}=\sqrt{g / l}\right)$ increases with the vertical acceleration $(g)$ and decreases with length $(l)$, the slosh mode natural frequency increases with vertical acceleration, and decreases with tank diameter. The natural frequencies of slosh modes in large diameters tanks with lower levels of acceleration can be easily be $0.5 \mathrm{~Hz}$ or lower.

For cylindrical tanks the slosh mass, location, natural frequency, and damping can be predicted using analytical and empirical relationships (Dodge [5]). Often sub-scale ground testing is done to validate or adjust predictions. Recently, Computational Fluid Dynamics (CFD) has also been used in conjunction with sub-scale testing to develop better predictions of slosh parameters. The specifics of the research into predicting slosh parameters is outside the scope of this paper. However, the key result is that the effective damping coefficient increases as a function of slosh motion magnitude due to increased fluid energy dissipation with larger motions.

R. A. Hall, Jr., is with CRM Solutions, Jacobs ESSSA Group, Huntsville, AL 35802 USA. (e-mail: robert.a.hall@nasa.gov).

J. F. Powers is with the National Aeronautics and Space Agency, Marshall Space Flight Center, Huntsville, AL 35812 USA, (e-mail: joey.powers@nasa.gov). 
The interaction of the slosh with vehicle control has been known to control system designers since the beginning of the space age in the 1960s. Due to large diameter tanks often used on larger launch vehicles, non-slosh considerations such as performance and structural dynamics, the primary attitude control frequency is often in proximity to slosh modes. One of the important results discussed in Bauer [2] and Greensite [3] is that for an attitude control system with attitude and attitude rate feedback (i.e. PD control) with a single dominant slosh mass, only slosh mass locations between the vehicle CG and the vehicle center of percussion are destabilizing. For more complicated vehicles with multiple tanks, some of whose frequencies are close enough proximity to dynamically couple, the result no longer fully holds, but through practice is a been shown to be a good guideline for which slosh tanks may need baffles for extra damping.

The traditional method for either accepting a given baffle design or for generating damping requirements for a baffle design is primarily based on control system stability analysis. The goal is to achieve the desired gain and phase margins of 6 $\mathrm{dB}$ and 30 deg respectively, which are the traditional margins used for launch vehicles (see Dennehy [4] and Frosch \& Vallely [1]). This can be done with a numerical search routine as describe in Orr [6]. The wave height, of which the slosh damping is a strong function of, is traditionally not used inside the control system analysis and is instead applied based on a fixed value when the baffle damping is computed.

The main impact to the control system in the event of unstable or poor slosh stability is the potentially excessive usage of the thrust vector control (TVC) system. For a space vehicle with liquid engines, the TVC system is usually composed of gimballed liquid engines which are pivoted by means of a hydraulic or electro-mechanical linear actuator. Limits on linear actuator travel or engine interference limits maximum TVC angle deflection, power limits maximum TVC angular rates, and energy storage and temperature limitation constrain the allowable duty cycle. Significant impacts to vehicle and crew survivability may occur if these limits are encountered.

The method presented in this paper is to augment the traditional linear approaches by using the non-linear damping profile as part of control system analysis. Central to the nonlinear analysis is the assessment of the impact to the TVC metrics resulting from a particular nonlinear slosh damping relationship. The method remains highly practical as a standard analysis method since it is still largely based upon linear-time-invariant (LTI) model, by computing several key transfer functions over a range of wave heights for a particular trajectory analysis point.

\section{SySTEM EQUATIONS}

\section{A. Plant Dynamics}

The systems equations of motions for a liquid rocket controlled by vectoring thrust $R^{\prime}$ at the rear of the vehicle through gimballed engines (see Figure 1), with $n$ sloshing tanks represented using the spring-mass-damper model are here presented. The equations are based on the linearized planar equations of motions presented in Frosch \& Vallely [1]

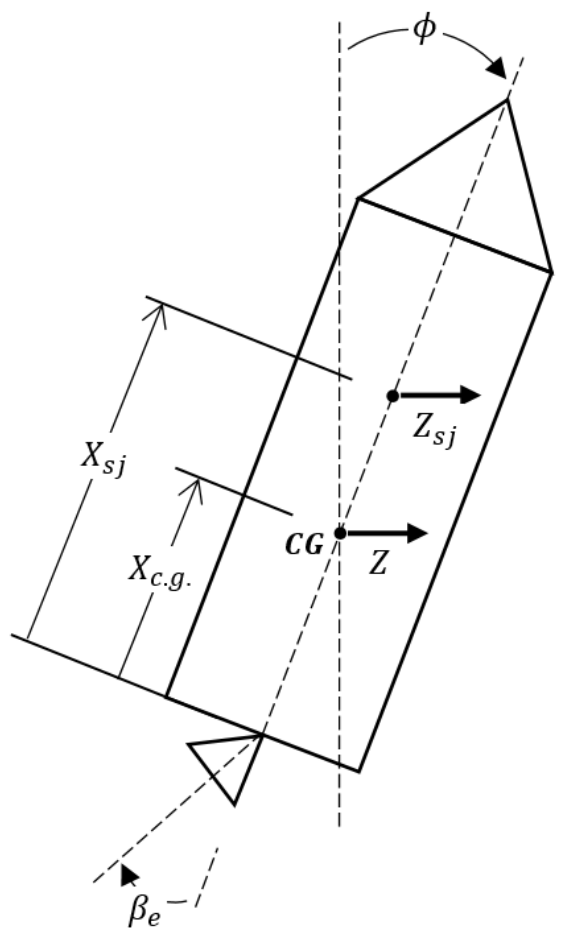

Figure 1. Space vehicle diagram with gimballed thrust vector and slosh mass.

which were used for control design and analysis of the Saturn V S-IC stage. Dynamics not needed which were removed to simplify the system include structural dynamics, aerodynamics, nozzle dynamics, and sensor dynamics. These dynamics are not needed in order to show the impact of slosh on stability, but when included these dynamics do constrain the control design sufficiently to often force the main control frequency to be in proximity to slosh modes.

$$
\begin{array}{r}
\phi s^{2}=-c_{2} \beta_{e}-\frac{1}{I} \sum_{j=1}^{n} m_{s j}\left(l_{s j} s^{2}+k_{3}\right) Z_{s j} \\
\mathrm{Zs}^{2}=k_{4} \beta_{e}+k_{3} \phi-\frac{1}{M} \sum_{j=1}^{n} m_{s j} z_{s j} s^{2} \\
\left(s^{2}+2 \zeta_{s j} \omega_{s j}+\omega_{s j}{ }^{2}\right) Z_{s j}=-\mathrm{Zs}^{2}+\left(l_{s j} s^{2}+k_{3}\right) \phi
\end{array}
$$

The transfer function equations of motion are converted to state space form, assuming only a single slosh mass. A matrix $E$ represents the coupled mass matrix and is numerically inverted prior to the computation of the final state space equations. The outputs of the state space equations used for control are the attitude angle and rate of the vehicle.

$$
\begin{aligned}
& \mathrm{x}=\left[\begin{array}{c}
\mathrm{z} \\
\phi \\
\mathrm{z}_{\mathrm{s} 1} \\
\dot{\mathrm{z}} \\
\dot{\phi} \\
\mathrm{z}_{\mathrm{s} 1}
\end{array}\right] \\
& \mathrm{u}=\beta_{\mathrm{e}}
\end{aligned}
$$




$$
\begin{aligned}
& \mathrm{E}=\left[\begin{array}{cccccc}
1 & 0 & 0 & 0 & 0 & 0 \\
0 & 1 & 0 & 0 & 0 & 0 \\
0 & 0 & 1 & 0 & 0 & 0 \\
0 & 0 & 0 & 1 & 0 & \frac{\mathrm{m}_{\mathrm{s} 1}}{\mathrm{M}} \\
0 & 0 & 0 & 0 & 1 & -\frac{\mathrm{m}_{\mathrm{s} 1} \mathrm{l}_{\mathrm{sj}}}{\mathrm{I}} \\
0 & 0 & 0 & 1 & -\mathrm{l}_{\mathrm{sj}} & 1
\end{array}\right] \\
& \mathrm{A}^{\prime}=\left[\begin{array}{cccccc}
0 & 0 & 0 & 1 & 0 & 0 \\
0 & 0 & 0 & 0 & 1 & 0 \\
0 & 0 & 0 & 0 & 0 & 1 \\
0 & \mathrm{k}_{3} & 0 & 0 & 0 & 0 \\
0 & 0 & \mathrm{~m}_{\mathrm{s} 1} \mathrm{k}_{3} / \mathrm{I} & 0 & 0 & 0 \\
0 & \mathrm{k}_{3} & -\omega_{\mathrm{s} 1}{ }^{2} & 0 & 0 & -2 \zeta_{\mathrm{s} 1} \omega_{\mathrm{s} 1}
\end{array}\right] \\
& \mathrm{B}^{\prime}=\left[\begin{array}{c}
0 \\
0 \\
0 \\
\mathrm{k}_{4} \\
-\mathrm{C}_{2} \\
0
\end{array}\right] \\
& C=\left[\begin{array}{llllll}
0 & 1 & 0 & 0 & 0 & 0 \\
0 & 0 & 0 & 0 & 1 & 0
\end{array}\right] \\
& E \dot{x}=A^{\prime} x+B^{\prime} u \\
& \dot{\mathrm{x}}=\left(\mathrm{E}^{-1} \mathrm{~A}^{\prime}\right) \mathrm{x}+\left(\mathrm{E}^{-1} \mathrm{~B}^{\prime}\right) \mathrm{u} \\
& \mathrm{y}=\mathrm{Cx}
\end{aligned}
$$

\section{B. Control System}

The control systems is simplified to be composed of attitude rate and attitude feedback only while sensor dynamics, actuator dynamics, internal delays, additional filtering for structural dynamics, and accelerometer feedback are omitted. The plant system and control system are connected to create the closed loop system. For open loop stability analysis, the loop is broken at the thrust vector angle $\beta_{e}$. Note that vehicle drift $(z, \dot{z})$ is not observable through the feedback variables, resulting in neutrally stable poles. Depending on the portion of flight, drift is either left un-controlled or is controlled through accelerometer feedback and/or a much slower outer guidance loop not analyzed here.

$$
\beta_{\mathrm{e}}=-\mathrm{a}_{0} \phi-\mathrm{a}_{1} \dot{\phi}
$$

\begin{tabular}{|c|c|c|}
\hline Name & Value & Units \\
\hline$F\left(=\mathbf{R}^{\prime}\right)$ & $9 \times 10^{5}$ & $\mathbf{N}$ \\
\hline$M$ & $1.6 \times 10^{4}$ & kg \\
\hline I & $9 \times 10^{5}$ & kg-m ${ }^{2}$ \\
\hline$X_{c . g}$ & 6 & $\mathbf{m}$ \\
\hline$X_{s 1}$ & 9 & $\mathbf{m}$ \\
\hline$m_{s 1}$ & $1.7 \times 10^{3}$ & kg \\
\hline$\omega_{s 1}$ & 2 & $\mathrm{rad} / \mathrm{s}$ \\
\hline$\zeta_{s 1}$ & $\begin{array}{c}\text { function of slosh } \\
\text { amplitude }\end{array}$ & - \\
\hline$a_{0}$ & 0.08 & $\mathrm{rad} / \mathrm{rad}$ \\
\hline$a_{1}$ & 0.14 & $\mathrm{rad} /(\mathrm{rad} / \mathrm{s})$ \\
\hline
\end{tabular}

\section{Example System Parameters}

A set of parameters were chosen to represent a hypothetical large upper stage similar in size to the upper stages used on the Saturn V or the Space Launch System. The parameters chosen exhibit poor slosh phase characteristics and proximal frequencies that readily challenge flight control stability.

TABLE I. SYSTEM PARAMETERS
A plausible damping vs. slosh displacement amplitude is described by (14). For an actual launch vehicle design, this profile would be a function of liquid level in the tank and any slosh baffles present, and would be arrived at through ground testing and CFD.

$$
\zeta_{\mathrm{s} 1}=10 \frac{1}{\mathrm{~m}} *\left(\text { oscillation amplitude of } \mathrm{z}_{\mathrm{sj}} \text { in } \mathrm{m}\right)
$$

\section{Non-Linear Damping Simulation Method}

Add method for simulation non-linear damping in time domain here.

\section{SLOSH LOCATION SENSITIVITY}

As discussed previously, the "danger zone" for slosh instability is, generally speaking, when the slosh mass is located between the mass center and center of percussion. The actual stability bounds can be derived by the equations of motion (Equations 1-3) and recognizing that the slosh phase instability will occur when, given the transfer function

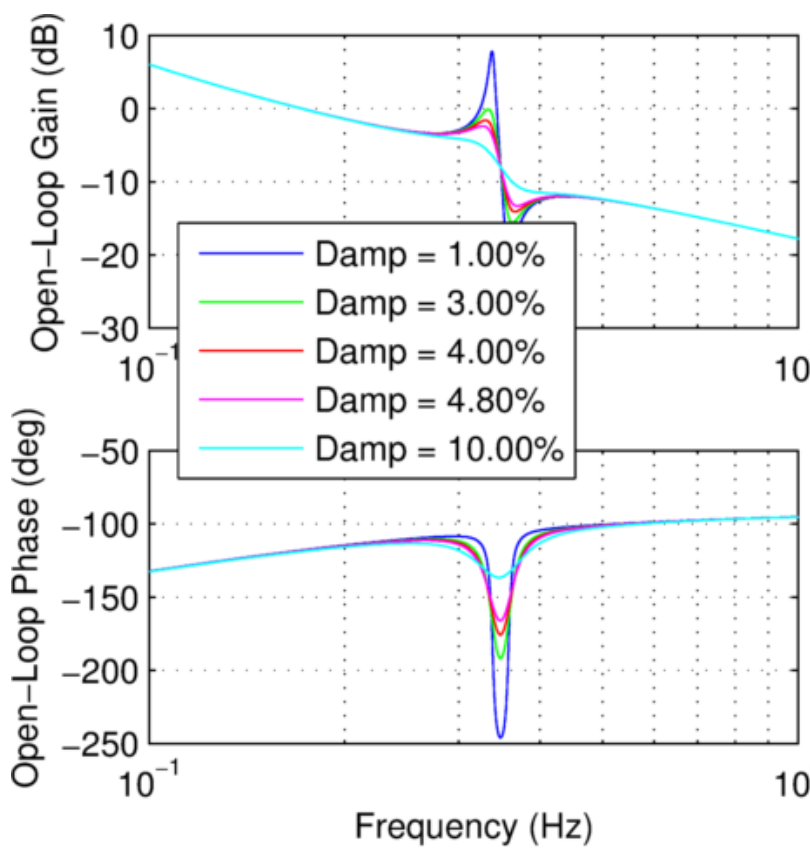

Figure 2. Bode of open loop response of example system with varying slosh mode damping. 
from vehicle gimbal to vehicle attitude, the zero associated with slosh dynamics is above the corresponding slosh pole.

Add full derivation here resulting in equations (15) and (16) for the range of $l_{s . j .}$. within the "danger zone".

$$
\begin{gathered}
l_{s . j .}<l_{c . p .} \\
l_{s j}>-\frac{F\left(M-m_{s j}\right)}{\left(M^{2} \omega_{s j}{ }^{2}\right)}
\end{gathered}
$$

\section{ANALYSIS METHOD}

\section{A. Stability Analysis}

An LTI system analysis can be performed on the system for different values of slosh damping. The Bode diagram of the open loop system is show in Figure 2. The slosh mode at approximately $0.35 \mathrm{~Hz}$ is at about twice the frequency of the $0 \mathrm{~dB}$ cross over frequency of $0.17 \mathrm{~Hz}$.

A Nichols diagram of the open loop response is shown in Figure 4 with a disc margin $(D M)$ threshold of 1 connecting the desired gain margin $(G M \mathrm{~d})$ of $6 \mathrm{~dB}$ and phase margin $(P M d)$ of $\frac{1}{6 \pi} \mathrm{rad}(30 \mathrm{deg}$ ) from the critical point at $0 \mathrm{~dB}$ and $\pi(-180 \mathrm{deg})$. The disc margin represents the radius of the response $(G(\omega))$ in a gain and phase margin normalized Nichols plot and is computed by equation (17). A minimum disc margin of 0.0 would correspond to a neutrally stable system and value of 1.0 would be minimum acceptable value for a robust control design.

$$
\mathrm{DM}=\sqrt{\begin{array}{l}
\left(\frac{20 \log _{10}(|\mathrm{G}(\omega)|)}{\mathrm{GMd}}\right)^{2}+ \\
\left(\frac{((\angle \mathrm{G}(\omega) \bmod 2 \pi)-\pi)}{\mathrm{PMd}}\right)^{2}
\end{array}}
$$

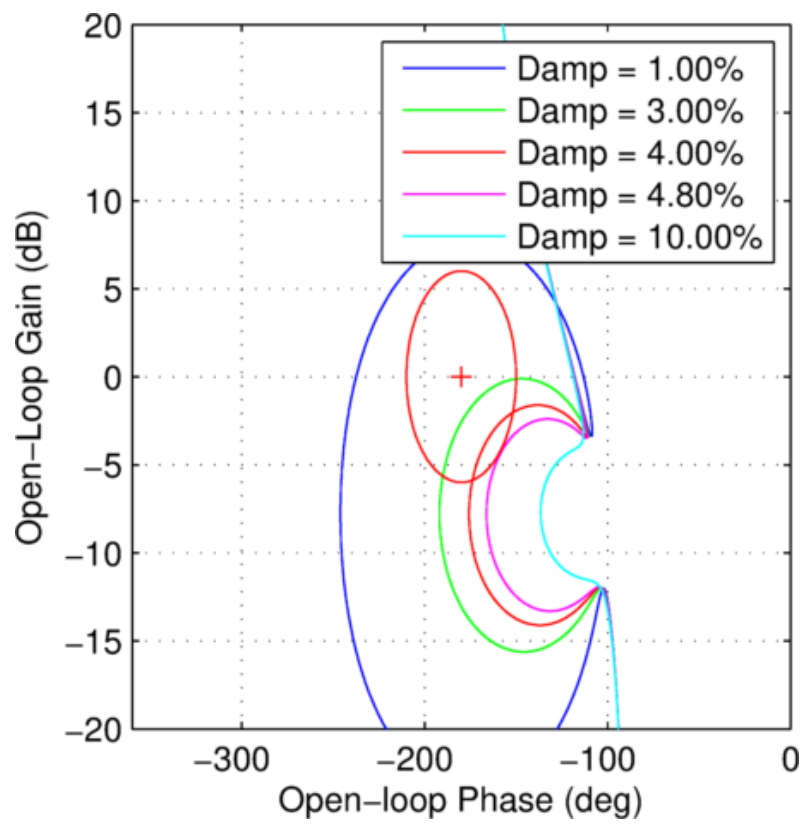

Figure 4. Nichols of open loop response of example system with varying slosh mode damping.

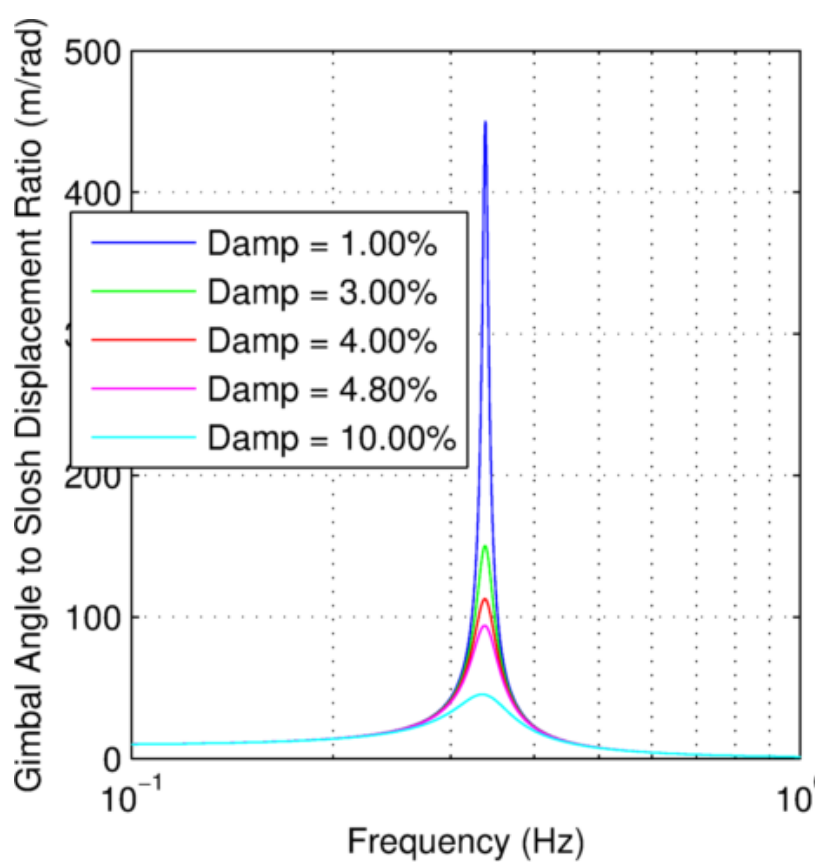

Figure 3. Thrust vector angle to slosh displacement gain of plant for varying slosh damping.

As can be seen in the Nichols diagram, the resulting LTI stability is a strong function of slosh damping and associated slosh amplitude. With a slosh mode damping of $1 \%$ the system is unstable. A damping of $4.8 \%$ corresponds to being right on the desired disc margin. The $3 \%$ damping response is an example where the gain and phase margins are ample but the system is not robust, showing the importance of using a combined metric such as the disc margin.

The presence of time delay, actuator dynamics, and filters to attenuate structural dynamics would have the effect of adding additional phase lag at the slosh frequencies and this effect is not represented in the example system. In the Nichols plot this would have the effect of bending the response to the left underneath the disc, sometimes making the slosh response come closer to the critical point from the bottom or from the bottom left.

\section{B. Thrust Vector Angle to Slosh Displacement Gain}

The gain of the transfer function from the thrust vector angle $\beta_{e}$ to the slosh mass displacement $z_{s j}$ from the plant dynamics are shown in Figure 3. Since this magnitude relationship is solely defined by the plant dynamics alone, it is not effected by any changes in the control system. As slosh damping decreases the gain of $z_{s 1} / \beta_{e}$ of increases. The consequence, is that for a given sized slosh limit cycle amplitude, a high gain means a small thrust vector angle amplitude. This simple fundamental relationship, along with the non-linear damping relationships enables the limit cycle assessment shown in the following section.

\section{Predicted Thrust Vector Amplitude Limit Cycle}

As demonstrated in Figure 3, for a given slosh damping profile the stability of the system is a function of amplitude of the slosh, with the system being unstable at very low 


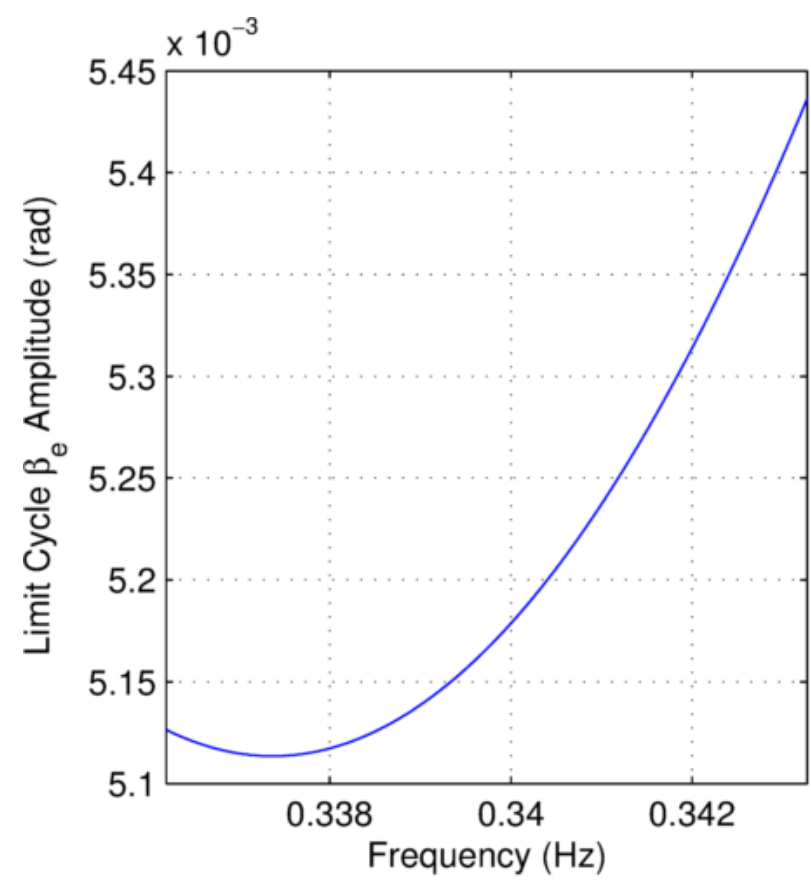

Figure 5. Predicted limit cycle amplitude of thrust vector angle over frequency range below $\mathrm{DM}_{\text {limit-cycle-threshold }}$ for example system with a slosh damping ratio of $4.8 \%$.

amplitudes, and stable at high amplitudes. For unforced conditions, a small unstable amplitude will grow in amplitude until damping is high enough that neutral stability is reached, resulting in a stable limit cycle. The damping corresponding neutral stability is associated with a slosh amplitude via the relationship in (14), and can be subsequently related to the thrust vector angle limit cycle amplitude by using the inverse of $\mathrm{z}_{\mathrm{s} 1} / \beta_{\mathrm{e}}$ transfer functions corresponding to the damping at neutral stability.

In order to properly account for margins in this process, the control system designer may choose both the permissible disc margin associated with the particular offending slosh mode as well as the acceptably small TVC limit cycle. The process is as follow: Find the damping which corresponds with just meeting threshold disc margin $\mathrm{DM}_{\text {damping-threshold }}$ (e.g. $4.8 \%$ damping for $\mathrm{DM}=1$ in the example system) and assume that the disc margin is degraded such that the system becomes neutrally stable. For time domain simulation, this could be accomplished with a gain change and time delay combination, but the method above is not explicitly dependent on the method or means by which the stability margin is degraded. For example, if the gain and phase degradation occurs outside of the thrust vector angle to slosh displacement dynamics, (e.g. sensor dynamics, flight control system dynamics, or actuator dynamics) then the thrust vector angle to slosh displacement gain won't be affected. If the degradation does occur inside the thrust vector to slosh dynamics then the thrust vector to slosh gain will change, but it is conservative to assume it is unchanged if slosh frequency greater than control frequency, since that situation involves a gain increase to cause the slosh mode to reach neutral stability, and that gain increase would correspond with a smaller TVC angle for a given slosh displacement. The other case, when slosh frequency is less than the control frequency, is not likely in launch vehicle control system designs since they are typically bandwidth constrained by the presence of structural dynamics at frequencies above the slosh frequencies.

The thrust vector angle to slosh displacement gain is a function of frequency and it is large close the slosh frequency and small further away. If limit cycle were to develop far away from the slosh frequency, for example at the rigid body gain cross over frequency, then slosh displacement will only play a very small part of that, and thus even high slosh amplitudes, and the subsequent increases in slosh damping will have little effect on the rigid body control mode. To select the frequency range of interest, a limit cycle threshold DM (DM $\mathrm{DM}_{\text {limit-cycle- }}$ threshold) value is chosen and all frequencies of the response which are below this threshold DM value are evaluated. To review the steps of the method are as follows:

1. Chose a TVC angle amplitude which is considered sufficiently small to be acceptable for degraded conditions.

2. Chose $\mathrm{DM}_{\text {damping-threshold and find the damping }}$ associated with being able to meet this margin. Determine the associated slosh displacement amplitude corresponding to this damping.

3. Chose $\mathrm{DM}_{\text {limit-cycle-threshold and find the slosh }}$ related frequencies below this margin.

4. Multiply the slosh displacement amplitude by the inverse of the $z_{s 1} / \beta_{e}$ transfer function for the determined frequency range to find the predicted limit TVC cycle amplitude under the degraded conditions.

5. Check the maximum limit cycle amplitude over the frequency range against the chosen TVC threshold.

For the example system, TVC limit of 0.5 degrees is chosen as an acceptably small limit cycle under degraded conditions, a $\mathrm{DM}_{\text {damping-threshold }}$ of 1 is chosen for finding the slosh damping which just meet a minimum disc margin, and a $\mathrm{DM}_{\text {limit-cycle-threshold }}$ of 1.1 is chosen for finding the slosh frequencies to use for evaluating the thrust vector limit cycle amplitude. If there were other dynamics further away from slosh, which have a lower disc margin than this threshold, then those frequencies can be eliminated based on a frequency threshold. In the example system, a damping of $4.8 \%$ (corresponding to a slosh amplitude of $0.48 \mathrm{~m}$ ) meets the $\mathrm{DM}_{\text {damping-threshold, and a frequency range between }}$ approximately 0.336 and $0.343 \mathrm{~Hz}$ corresponds to a disc

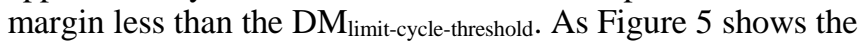
predicted limit cycle amplitude over the frequency range is on the order of 5 milliradians or approximately 0.3 degrees.

The limit cycle prediction can easily be tested by degrading the gain and phase of the nominal system at the $4.8 \%$ damping level. Taking the minimum DM point at $0.34 \mathrm{~Hz}$ and degrading the control system with gain of $4.4 \mathrm{~dB}$ and a time delay of 170 ms results in a system with neutrally stable complex poles at that frequency. A LTI impulse response of the neutrally stable system is shown in Figure 6. As can be seen, for a slosh displacement of $0.48 \mathrm{~m}$, a TVC angle amplitude of about 0.005 radians results. 

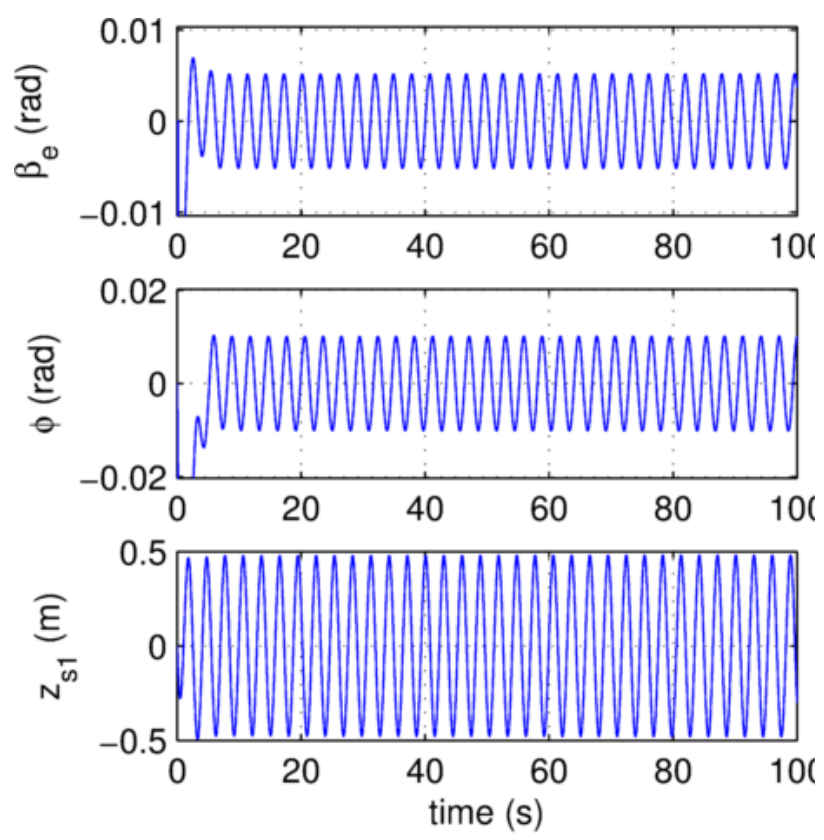

Figure 6. Time domain limit cycle demonstration of example system with a slosh damping of $4.8 \%$ and degraded gain and phase.

\section{Non-Linear Simulation Results}

Add non-linear simulation results here.

\section{CONCLUSION}

The analysis method presented herein is a method for accepting the control system stability characteristics of a liquid propelled space vehicle given a model of slosh damping as a function of slosh displacement magnitude. The analysis method can also be used to develop a damping requirements profile that can be used to design the needed slosh baffles for a new design. The values of the TVC threshold, DM $_{\text {damping- }}$ threshold and $\mathrm{DM}_{\text {limit-cycle-threshold }}$ are a choice to the control system designer. The values chosen for the example system $(0.5$ degrees, 1.0, and 1.1) are considered a reasonable conservative set of values. The disc margin values can be argued to be over-conservative since for other dynamics, such as aerodynamics or rigid body dynamics, a DM of 1.0 is considered acceptable for a nominal system, and there is no expectation that a limit cycle of sufficiently small consequence would occur should the full DM be lost.

It is important to stress that the above analysis treats only stability and not vehicle performance during external disturbances. A non-linear Monte Carlo analysis with the non-linear damping profile and the appropriate external forcing functions should also be done to ensure acceptable performance. It is possible that the forced response will drive the slosh displacement high enough that it is outside of the range that has been characterized and can be accurately modelled or high enough to cause crash over and droplet formation which increases heat transfer from a cryogenic liquid to the pressurization gas in the ullage space above the liquid.

\section{ACKNOWLEDGMENT}

We acknowledge John Wall for many discussions relating to this topic.

\section{REFERENCES}

[1] J. Frosch and D. Vallely, "Saturn AS-501/S-IC Flight Control System Design" in AIAA Journal of Spacecraft, vol. 4, No. 8, Aug. 1967, pp. 1003-1009.

[2] H. F. Bauer, "Stability Boundaries of Liquid-Propelled Space Vehicles with Sloshing" in AIAA Journal, vol. 1, No. 7, Jul. 1963, pp. 1583-1589.

[3] A. L. Greensite, "Analysis and Design of Space Vehicle Flight Control Systems - Volume VII - Attitude Control During Launch N67-34031", General Dynamics Corporation, Jul. 1967, pp. 41-46.

[4] C. J. Dennehy, K. Lebsock, J. West, GN\&C Engineering Best Practices For Human Rated Spacecraft Systems, NASA/TM-2008215106, Jan. 2008

[5] F. T. Dodge, The New "Dynamic Behavior of Liquids in Moving Containers", Southwest Research Inst., San Antonio, 2000, URL https://books.google.com/books?id=RltitwAACAAJ

[6] J. Orr, C. Hall, "Parametric Optimization of Ares I Propellant Slosh Characteristics Using Frequency Response Criteria", AIAA Guidance, Navigation, and Control Conference, Aug. 2009

[7] B. Bjelde, M. Vozoff, G. Shotwell, "The Falcon 1 Launch Vehicle: Demonstration Flights, Status, Manifest, and Upgrade Path", SSC-07III-6, $21^{\text {st }}$ Annual AIAA/USU Conference on Small Satellites, 2007 